\title{
Can pelvic measurements predict gender identity disorder?
}

Surgeons and anthropologists from the University of Lodz, Poland, have collaborated on a recent study to show that female-to-male (FtM) transsexuals display certain male-typical pelvic characteristics, indicating a biological basis for gender identity disorder. Sitek et al. compared 17 pelvic X-ray measurements from $24 \mathrm{FtM}$ transsexuals and 48 age-matched controls (24 males and 24 females) - all of whom presented for X-ray with pelvic pain, but no evidence of pelvic injury or history of pelvic trauma (or pregnancy beyond 3 months). The FtM transsexuals included in the study began hormonotherapy at a mean age of 21.6 years. At the time when $\mathrm{X}$-rays were recorded, they had been taking hormones for a mean period of 4.9 years.

The pelvis is the most dimorphic structure in the human skeleton. Gender identification based on a single pelvic bone (the pubic bone) yields a sex determination accuracy of up to $95 \%$. In the study by Sitek and colleagues, seven pelvic features were identified as larger in

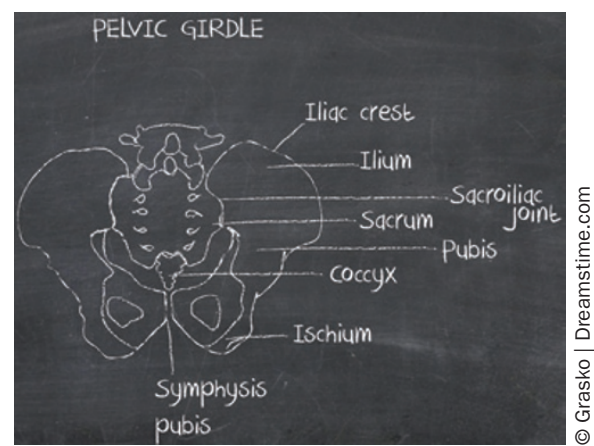

pubic symphysis height and breadth, and ischium height). Right obturator foramen length was significantly larger for transsexuals than for either control group. Interestingly, the female-typical features that were retained in FtM transsexuals (for example, pelvis height) are known to have less impact on reproductive function than most other pelvic variables.

From these data, the authors developed a model for distinguishing FtM transsexuals from male and males than in females (including pelvis height), whereas nine features were bigger in females (including greatest pelvis breadth). Only one feature (right obturator foramen length) was not distinguishable between the two groups.

FtM transsexuals displayed maletypical measurements for six pelvic features (femoral neck-shaft angle, anatomical conjugate, greatest pelvis breadth, interacetabular distance, pelvic inlet breadth, and upper breadth of the sacrum) and intermediate measurements for three pelvic features (subpubic angle, female controls using a combination of four variables; the height of the pubic symphysis, the greatest pelvic breadth, the interischial distance, and the acetabular diameter. Application of this model led to a high proportion of correctly identified cases, suggesting potential value in a clinical setting.

Melanie Clyne

Original article Sitek, A. et al. Biometric characteristics of the pelvis in female-to-male transsexuals. Arch. Sex. Behav doi:10.1007/s10508-012-9989-4 